\title{
Epithelial-mesenchymal transition-associated microRNAs in colorectal cancer and drug-targeted therapies (Review)
}

\author{
DANJUAN JIN*, YANTIAN FANG* , ZHENGYANG LI, ZONGYOU CHEN and JIANBIN XIANG \\ Department of General Surgery, Huashan Hospital, Fudan University, Shanghai 200040, P.R. China
}

Received July 2, 2014; Accepted November 17, 2014

DOI: $10.3892 /$ or.2014.3638

\begin{abstract}
Colorectal cancer (CRC) is one of the most common malignancies, and the third leading cause of cancer-associated mortality worldwide. Therefore, the identification of effective targets at the early stages of cancer invasion and metastasis, and new tumor markers for early diagnosis and individualized treatment is imperative for CRC. Epithelial-mesenchymal transition (EMT) refers to the phenomenon of the transformation of epithelial cells to mesenchymal cells in specific physiological and/or pathological circumstances. Evidence suggests that EMT plays an important role in in situ infiltration and distant metastasis of many types of cancer, including CRC. Recent findings showed that microRNA expression is important in regulating the EMT process. This review aimed to summarize EMT-associated specific miRNA molecules in $\mathrm{CRC}$, with particular emphasis on clinical targets for effective treatment of this lethal disease.
\end{abstract}

\section{Contents}

1. Introduction

2. EMT and cancer

3. EMT-associated microRNA in colorectal cancer

4. Strategies for therapy targeting EMT in colorectal cancer

5. Conclusion and perspective

\section{Introduction}

Colorectal cancer (CRC) is one of the most common malignancies, and the third leading cause of cancer-associated

Correspondence to: Dr Zongyou Chen or Dr Jianbin Xiang, Department of General Surgery, Huashan Hospital, Fudan University, No. 12 Middle Wulumuqi Road, Shanghai 200040, P.R. China

E-mail: czyshhs@126.com

E-mail:xjbzhw@163.com

*Contributed equally

Key words: epithelial-mesenchymal transition, microRNA, colorectal cancer mortality worldwide (1). Although a decrease in CRC mortality has been observed, its incidence continues to rise. Therapeutic approaches such as conventional surgery, chemotherapy and radiation therapy are insufficient with regard to reversal of advanced CRC. Thus, identification of new tumor markers for early diagnosis and therapy, is the key to reducing the incidence of cancer, directly affecting the prevention and treatment of CRC.

Epithelial integrity is crucial during epithelial tumor development. Eradication of the original epithelial structure and obtaining the peculiarity of mesenchymal cells is the first step in tumor invasion and metastasis. This procedure is known as epithelial-mesenchymal transformation (EMT), which plays an important role in in situ infiltration and distant metastasis in various types of cancer, including CRC (2).

MicroRNAs (miRNAs) are small non-coding RNA molecules, consisting of 22-25 nucleotides, that regulate the expression of target genes through base-pairing interactions. These molecules are important in tumorigenesis including angiogenesis, circulation, extravasation, intravasation, invasion and metastatic colonization (3). Studies have focused on tumor-associated miRNAs with regard to the expression and function of miRNAs.

This review aimed to summarize EMT-associated miRNA molecules in CRC to determine new types of treatment, particularly drug-targeted therapy.

\section{EMT and cancer}

EMT-associated concepts. Epithelial and mesenchymal cell types are recognized by their unique cell morphology and peculiarity in tissues. Epithelial cells belong to a group of polarized cells that are connected laterally via adhesion molecules and cellular junctions, including adherent junctions, desmosomes, and tight junctions. By contrast, mesenchymal cells enable themselves to move freely in the extracellular matrix (ECM), without adhesion molecules or junctions (4). In many environments, cells change their own characteristics from epithelial-like to mesenchymal-like and vice versa, particularly in the period of embryonic morphogenesis processes. EMT is a biological process in which epithelial cells lose their polarities, intercellular junctions and epithelial-like characteristics, acquiring a less-differentiated phenotype with motile behavior. The entire process of EMT includes two aspects of cell morphology and genotype changes. It involves molecular 
reprogramming of the cell, including loss of cell adhesion molecules such as E-cadherin; changing shape of cells from epithelial morphology to the spindle shape of fiber cells and cell keratin structure; and acquiring some characteristics of mesenchymal cells or fibroblasts, such as overexpression of vimentin, N-cadherin, osteopontin, Snail, slug and other interstitial proteins (5-7). Thus, cells obtain higher abilities with regard to migration, invasion, antiapoptosis and the degradation of extracellular matrix. For these reasons, EMT is crucial during the early stages of invasion and metastasis of epithelial tumors (8-9).

Molecular markers in EMT. EMT is a dynamic process comprising a number of steps and complex regulatory mechanisms, including loss of adhesion between cells, destruction of the basement membrane (BM) and ECM, reconstruction of the cytoskeleton and enhancement of migration and motility (10). Thus, the molecular mechanisms and signaling pathways involved in EMT are mainly associated with the above aspects.

$E$-cadherin/ $\beta$-catenin complex. In the process of embryonic development, cellular adhesive molecular regulation follows the principle of specific time and space, of which cadherin is the most important. E-cadherin is a calciumdependent transmembrane glycoprotein on the cell surface that mediates cell-cell adhesion, which can form the E-/ $/ \beta-/ \alpha-$ catenin complex by binding to $\beta$-catenin inside the cytoplasm . The complex connects directly to the actin cytoskeleton to maintain the stability of intercellular adhesion and polarity, maintaining epithelial cell integrity and normal function (11). Thus, loss of E-cadherin expression or conversion between different cadherin proteins may induce EMT. At the same time, decreasing E-cadherin expression can increase $\beta$-catenin in the cytoplasm, which binds to transcription factors TCF/LEF. Loss of E-cadherin expression in human tumors is most commonly caused by methylation of its promoter (12), phosphorylation and degradation of protein (13), and upregulation of the transcriptional repressors Snail (SNAI1), Slug (SNAI2), Sip1 (GEMIN2) and Zeb1, targeting the E-cadherin promoter (14-15). In the epithelial tumors, EMT cleaves cancer cells from the primary focal, invades the blood vessels or lymph nodes and migrates. Thus, E-cadherin is an important factor in tumor malignant transformation, invasion and metastasis.

$\mathrm{N}$-cadherin. $\mathrm{N}$-cadherin is s skeleton protein present in mesenchymal cells, which is expressed in nerve, musculoskeletal and hematopoietic tissue, but not in normal epithelial tissue (16). Its main function involves the induction of fibers of cell dynamic adhesion, leading to mesenchymal cell migration. Hazan et al suggested that conversion occurs between cadherins. Expression of E-cadherin was reduced while that of $\mathrm{N}$-cadherin was increased, an important mechanism of EMT (17). Epithelial tumor cells therefore cleave from the primary focal, invade surrounding tissues, and migrate through lymphatic and blood circulation when E-cadherin expression is reduced while $\mathrm{N}$-cadherin expression is increased (18).

Vimentin. Vimentin is an intermediate filament protein derived from mesenchymal cells, that is hardly expressed in normal epithelial tissue. Abnormal expression of vimentin results in the reconstruction of cytoskeletal proteins, leading to epithelial cells acquiring some characteristics of spindle-like fibroblasts, which migrate more readily. Findings of previous studies have shown the abnormal expression of vimentin in a variety of epithelial tumors such as ovarian, breast, colonic and prostate cancer, which is closely associated with cancer invasion and metastasis (19-22).

Fibronectin. Fibronectin is an extracellular matrix glycoprotein, consisting of two disulfide-bound polypeptide molecular chains, that is mainly present in mesenchymal cells of normal tissue. Fibronectin contains a different functional and structural domain, that binds to the cell surface. In previous studies it was shown that, fibronectin increases migration in vivo and enhances the effect on cell adhesion and spreading. Fibronectin has been shown to play a role in cell morphology, cytoskeletal organization, phagocytosis, embryonic differentiation and wound repair (23-24), which are associated with tumor invasion and metastasis.

Transcription factors. Three core groups of transcriptional factors have been consistently identified to be critical during various EMT events, and are considered the core EMT regulators. The first group is the Snail zinc-finger family, including Snaill and Snail2, both of which directly bind to the E-boxes of the E-cadherin promoter to repress its transcription (25-26). The second group is the distantly associated zinc-finger E-box-binding homeobox family of proteins Zeb1 and Zeb2, which are also able to suppress E-cadherin transcription via a double-negative feedback loop controlling Zeb1/Zeb2 and miRNA-200 family expression (27-28). The third group is the basic helix-loop-helix (bHLH) family, including Twist1 and Twist2. Twist1 can repress E-cadherin through the induction of Snail transcription factors (29-31).

Signaling pathways and mechanisms involved in CRC EMT. EMT is a dynamic process, involving the phenotypic changes in protein, as an external adjustment. It is also associated with a variety of signal transduction pathways. Signal codes specifically bind to cell membrane surface receptors, activating transcription factors in the nucleus through a variety of signal transduction pathways (Fig. 1).

Wnt/ $\beta$-catenin/LEF-1 signaling pathway. The Wnt gene was first identified and reported in 1982 (32). This gene forms a complex signaling pathway in tumor cells, including at least three branches of the Wnt/ $\beta$-catenin/LEF-1 pathway, Wnt/ the planar cell polarity (PCP) pathway and the $\mathrm{Wnt} / \mathrm{ca}(2+)$ mediated pathway (32-34). The $\mathrm{Wnt} / \beta$-catenin pathway, known as the 'classic Wnt pathway', plays a role in the process of EMT in CRC (35). The Wnt/ $\beta$-catenin/LEF-1 signaling pathway is closely associated with EMT, which comprises the Wnt protein, frizzled protein, dishevelled protein, APC compounds, GSK- $3 \beta$, axin protein, $\beta$-catenin and TCF/LEF family of transcription factors. In tumors, due to abnormal activation, the Wnt gene binds to the frizzled protein through the Wnt ligands, forming a receptor complex with LRP5/6, inducing GSK-3 $\beta$ inactivation. APC, an axin protein is activated in succession, forming the GSK3 $3 / \mathrm{APC} /$ axin complex, and reducing the phosphorylation degradation of $\beta$-catenin. Thus, large amounts of $\beta$-catenin gather in the cytoplasm and nucleus, interacting with TCF/LEF transcription factors, and inducing the transcription of target genes of Wnt, such as TNC. Phosphorylation of tyrosine in the $\beta$-catenin extremity can lead to E-cadherin/ $\beta$-catenin complex decomposition, activating the Wnt signaling pathway (36-40). Thus, interaction between 


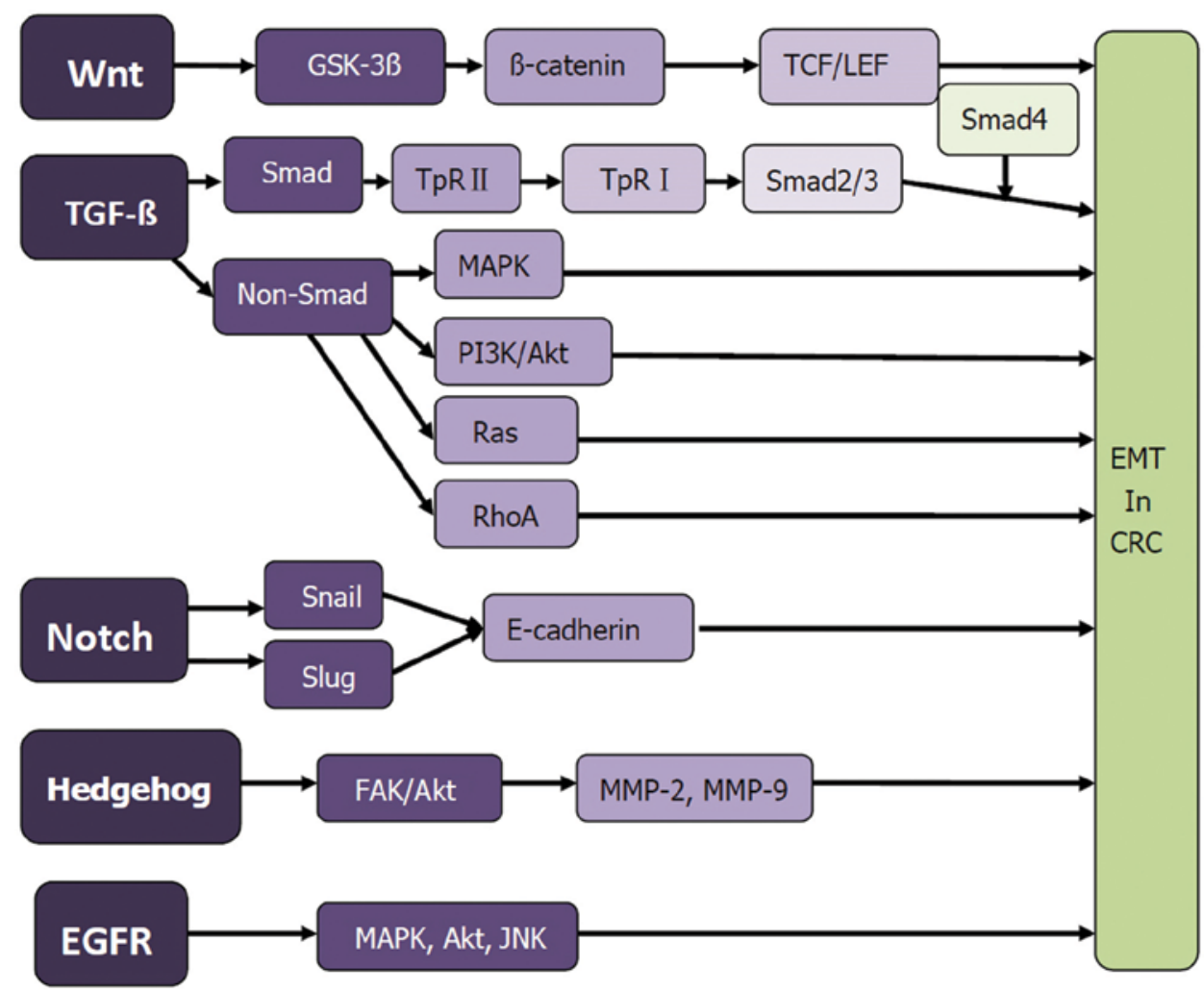

Figure 1. Signaling pathways involved in the epithelial-mesenchymal transition of colorectal cancer.

the Wnt pathway and the E-cadherin/ $\beta$-catenin complex potentially promotes tumor development.

TGF- $\beta$ signaling pathway. The transforming growth factor- $\beta$ (TGF- $\beta$ ) signaling pathway appears to be a primary inducer of EMT (41). Classic SMAD and other non-SMAD pathways participate in the EMT process induced by TGF- $\beta$ $(41,42)$. In the early stage of tumor growth, TGF- $\beta$ induces growth arrest and apoptosis as a tumor suppressor. In advanced stages of tumor progression, TGF- $\beta$ regulates transcription through SMAD-dependent/-independent TGF- $\beta$-receptor signaling pathways, initiating cancer growth and metastasis.

SMAD pathway. SMAD and signal-regulating proteins downstream of TGF- $\beta$ receptor complexes, are present in the cytoplasm, conducting signals from the cell membrane to the nucleus, and regulating gene transcription. A typical SMAD-dependent pathway involved in the EMT process is described below. TGF- $\beta$ ligand binds to the type 2 TGF- $\beta$ receptor, which recruits the type 1 TGF- $\beta$ receptor. This receptor dimerization and phosphorylation of serine/threonine residues allows the phosphorylation of SMAD2 and SMAD3. Activated SMAD proteins dissociate from the SMAD anchor for receptor activation (SARA) protein, hetero-oligomerize with SMAD4, and translocate to the nucleus, combining with the SMAD binding element located in EMT-associated gene promoter regions. This process mediates target gene expression or repression, such as MMP-2, SPI- 1 and $\alpha$-SMA. As a tumor-suppressor gene, SMAD4 increased epithelial marker expression of E-cadherin, strengthening the connection between cells in CRC and SW480 (7,43-45).

Non-SMAD pathway. TGF- $\beta$ signaling occurs through a number of non-SMAD pathways, including branches of
MAPK, RAS and PI3K/Akt. The main MAPK signaling pathways involved in EMT include the JNK signaling, p38 MAPK and Ras/Raf/MEK/ERK pathways. MAPK is mainly involved in the regulation of cell proliferation, secretion and neuronal differentiation. In the PI3K/Akt pathway, PI3K is activated by Ras combining to P110 or interacting with growth-factor receptors with phosphorylated tyrosine residues or junction protein. Akt activates or inhibits downstream target proteins by phosphorylation, regulating cell proliferation, differentiation, apoptosis and migration. In addition to PI3K/Akt, RAS, and MAPK, TGF- $\beta$ activates RhoA, c-Src, m-TOR, and protein phosphatase 2A (PP2A)/p70s6K, inducing EMT occurrence (46-51).

Notch signaling pathway. The Notch signaling pathway has been considered a traditional pathway regulating embryogenesis and tissue formation. However, it is involved in the occurrence of EMT. Notch can induce tumorigenesis, causing epithelial cells to lose their polarity, mobility and invasive ability (52). In this process, Notch regulates the expression of E-cadherin by inducing Slug activation or regulating Snail expression, thus enhancing the ability of tumor cell invasion and migration $(53,54)$. Notch signaling pathway does not function independently, but may combine its action with other signaling pathways to induce EMT (55).

Other signaling pathways. Studies have also shown that the Hedgehog (Hh) signaling pathway and epidermal growth factor receptor (EGFR) signaling cascades are involved in the EMT process. The Hh signaling pathway is crucial in embryonic development, formation and maintaining of cancer stem cells (CSCs) and EMT. Binding Hh ligands, such as Snoic, Desert, and Indian to Patched, results in de-repression 
of smoothened (SMO) and its activation, internalization and translocation to the primary cilium. The transcription of GLI target genes is followed by the activation of zinc-finger transcription factors GLI-1, GLI-2 and GLI-3 (56). The Hh signaling pathway induces cancer-cell invasion by regulating FAK/AKT signaling pathway-mediated activation, expressing MMP-2 and MMP-9 and inducing the expression of E-cadherin, Snail (57). As mentioned above, the EMT process is associated with ECM, otherwise, EGFR expression or activity may lead to cancer (58). Several auto-phosphorylation tyrosine residues in the C-terminal domain of EGFR elicits are activated by other proteins, principally the MAPK, Akt and JNK pathways (59). This stimulation promotes EMT and modulates phenotypes such as cell proliferation, migration and adhesion.

\section{EMT-associated microRNA in colorectal cancer}

MicroRNAs (miRNAs) are small non-coding RNAs regulating target mRNAs through post-transcription. Acting as endogenous suppressors, miRNAs inhibit gene expression through the imperfect binding of RISC subsequent to transcription. The main aim of miRNA is the degradation or translational inhibition of target mRNA (60). Therefore, miRNA affects cell processes, including cell differentiation, proliferation, metabolism and apoptosis by regulating one or two key target genes (61). miRNAs participate in tumor progression by acting as promoters or suppressors. miRNAs are also important in the regulation of cancer EMT, including CRC.

\section{miRNA and cancer EMT}

miRNA. miRNA was initially identified lin-4 in caenorhabditis elegans (62). However, extensive studies on miRNA were initiated following the identification of Let-7 (63). miRNAs are highly conserved, with tissue- and sequence-specific expression (64-66). The formation of mature miRNA binds to the imperfect complementary site of target mRNA via base pairing. When miRNA is fully complementary to the target site, the combination of these miRNAs often leads to the degradation of target mRNA through a RISC complex. When the miRNA and target mRNA sequences degree of matching is low, the translation process is inhibited $(67,68)$. miRNAs have been associated with various types of cancer. Approximately $50 \%$ of miRNAs in the genome were found to bind to fragile sites in tumors (69), suggesting that miRNAs play a critical role in tumorigenesis and metastasis, including the process of EMT.

miRNAs in cancer EMT

miRNAs act as tumor suppressors in EMT. In 2007, Hurteau et al found that the expression of miRNA-200c was negatively associated with E-cadherin expression in breast cells (70). miR-200 family and miR-205 were found to regulate EMT through inhibition of Zeb1 and Zeb2 E-cadherin $(71,72)$. In another study, TGF $\beta 2$ and Zeb1 were identified as the main targets of miR-200 family and miR-205. These findings indicated a feed-forward loop showing Zeb1 and miR-200 members to be involved in EMT and cancer cell invasion (73). Findings of recent studies have shown that Let-7 is associated with cancer EMT and metastasis. Let-7 was identified as targeting HMGA2, indicating there was a relationship between miRNA Let-7 and EMT (74). Downregulation of miRNA-138 is associated with interstitial cell-like transformation, and can enhance the metastasis and invasion of tumor cells including EMT with a decreased expression of E-cadherin, and enhanced expression of vimentin (75). Newly identified miRNAs, including miR-23b, miR-29c, miR-203, miR-448 and miR-194, similarly inhibited the EMT process (76-78).

miRNAs act as oncogenes in EMT. Overexpression of miR-10b, upregulated by EMT transcription factor Twist, located in the HOX gene cluster, is associated with invasiveness and metastatic potential in breast cancer (79). Results of studies, showed that miR-155 expression was increased in TGF-induced EMT in tumors, by SMAD4-regulated transcription activity. Breast cancer cells with miR-155 downregulation did not effectively induce EMT (80). In addition, miR-21 can promote the breast cancer EMT through overexpression of the SMAD-dependent transcription regulation matrix. By suppressing TIAM1 expression, miR-31 significantly enhanced CRC cell migration. It also induced cancer cell EMT process by activating transcription factors AP1 and Zeb1, located downstream of the TGF- $\beta$ signaling pathway (81-83). miR-9, miR-661, miR-369-5p, miR-370, miR450a and miR-542-5p also promoted EMT tumor (84-86).

miRNA regulation of EMT in CRC. miRNAs are involved in the CRC EMT, partly by regulating the expression of oncogenes and tumor suppressors and partly by functioning as oncogenes or tumor suppressors themselves (87). These important regulatory functions of miRNA-activated upstream factors, promoted the CRC EMT process. (Fig. 2)

miR-200 family. As mentioned earlier, the miR-200 family (miR-200a, miR-200b, miR-200c, miR-141, and miR-429) plays a key role in cancer EMT, including CRC. miR-200 family members were found to enhance E-cadherin expression by targeting complementary sites in the 3-UTR of Zeb1 and Zeb2. Loss of the basement membrane (BM), EMT was considered a key step in induction of metastasis. Zeb1 was found to be the key transcriptional repressor of BM components in CRC $(88,89)$. In addition, Zeb1 inhibits cell polarity factors and $\mathrm{Lgl} 2$ expression in CRC, which is critical for the epithelial phenotype (90). Transforming growth factor- $\beta$ (TGF- $\beta$ ), an EMT activator, is produced by tumor cells, triggering the expression of Zeb1/2, an upstream master regulator of EMT progression. Zeb1 directly suppressed the expression of miR-141 and miR-200c (on human chromosome 12), downregulating some essential features of EMT. After knockdown of Zeb1 in CRC, an increased expression of miR-141 and miR-200c can induce the epithelial phenotype, increase cell-cell adhesion, induce E-cadherin expression, and reduce cell migration and invasion (73). In addition, second miRNA clusters (miR-200b, miR-200A, and miR-429) of the miR-200 family also contain highly conserved putative Zeb1 binding sites in the upstream sequence (91). Results of those studies showed that, Zeb1 can trigger a miRNA-mediated feed-forward loop stabilizing the EMT and promoting cancer-cell invasion. Otherwise, strong induction of miR-200 may promote the differentiation and inhibition of epithelialmesenchymal-specific gene expression, by downregulating Zeb1 and Zeb2 conversely. Presumably, Zeb and the miR-200 family participate in a double-negative feedback loop stabilizing differentiation along the EMT-MET axis (92). 


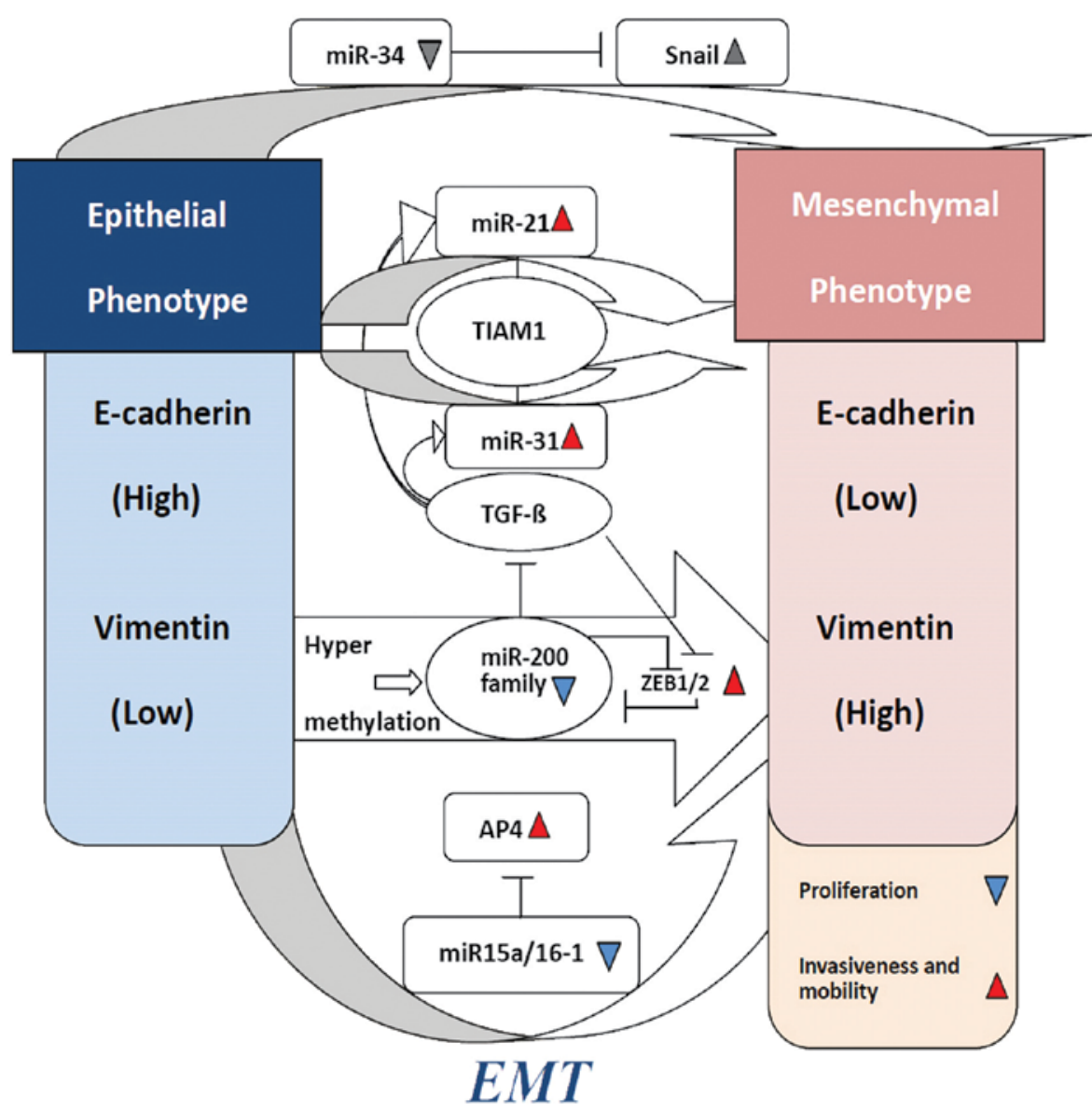

Figure 2. Regulation of epithelial-mesenchymal transition by miRNAs in colorectal cancer. Several miRNAs have been shown to directly or indirectly regulate the activities of cell phenotypic transition.

$m i R-21$ and $m i R-31$. miR-21 is located on human chromosome 17q23.2. In CRC, this genomic region always shows copy number gain, which is frequently observed in metastatic tumors. This observation suggests miR-21 is important in the CRC metastatic pathway. miR-21 encodes a single hairpin and is regulated by its own promoter containing binding sites for transcription factors, such as AP-1 and PU.1 (93). Upregulation of TGF- $\beta 1$ increased the expression of miR-31, resulting in a higher percentage of cells using a 'spreading' morphology. The levels of miR-21 and miR-31 were markedly elevated under the synergistic actions of TGF- $\beta / T N F-\alpha$. The two miRNAs are likely to have a number of different direct targets. They converge on TIAM1, a guanine nucleotide exchange factor (GEF) for the Rac GTPase-regulating migration and invasion of various cancer cells including CRC cells (81). It has been identified that, high miR-21 with low Integrin- $\beta 4$ (ITG $\beta 4$ ) can be exclusively expressed in polarized epithelial cells, and the level of PDCD4 expression was able to regulate EMT in CRC (94).

$m i R-34$. The miR-34 family includes miR-34a, miR-34b and miR-34c. The miR-34 family participates in cell cycle progression, senescence and cell apoptosis (95-97). The members inhibit EMT by downregulating the expression of Snail, one of the EMT-inducing transcription factors (EMT-TF) $(98,99)$. It has been revealed that IL-6 induces EMT, invasiveness, and the metastatic properties of CRC cells. By directly repressing miR-34a, miR-34a can suppress tumor progression by inhib- iting the IL-6R/STAT3/miR-34a feedback loop. Thus it can promote and maintain EMT, invasiveness, and metastasis in CRC (100).

miR-15a/16-1. miR-15a and miR-16-1 are located on human chromosome 13q14.3, which is highly conserved (101). Emerging evidence has shown that they target the 3'-untranslated region (3'-UTR) of the transcription factor AP4, which is downregulated by $\mathrm{p} 53$. AP4 directly represses E-cadherin via a non-canonical AP4-binding motif and induces $\mathrm{N}$-cadherin, mediating EMT in CRC. This finding suggests that miR-15a/16-1 and AP4 are involved in a double-negative feedback loop, stabilizing epithelial and mesenchymal states. Respectively, they may determine metastatic prowess in CRCs $(102,103)$.

\section{Strategies for therapy targeting EMT in colorectal cancer}

Improved understanding of the pathology of molecules in cancer had led to the development of a number of target drugs, which have demonstrated improved outcome of cancer patients with metastasis. However, the aim of ongoing animal experiments and clinical trials is to identify further drugs (Fig. 3).

Targeting EMT-associated molecules and pathways in CRC. As shown in Fig. 1, EMT-associated pathways provide therapy targets for CRC patients. 

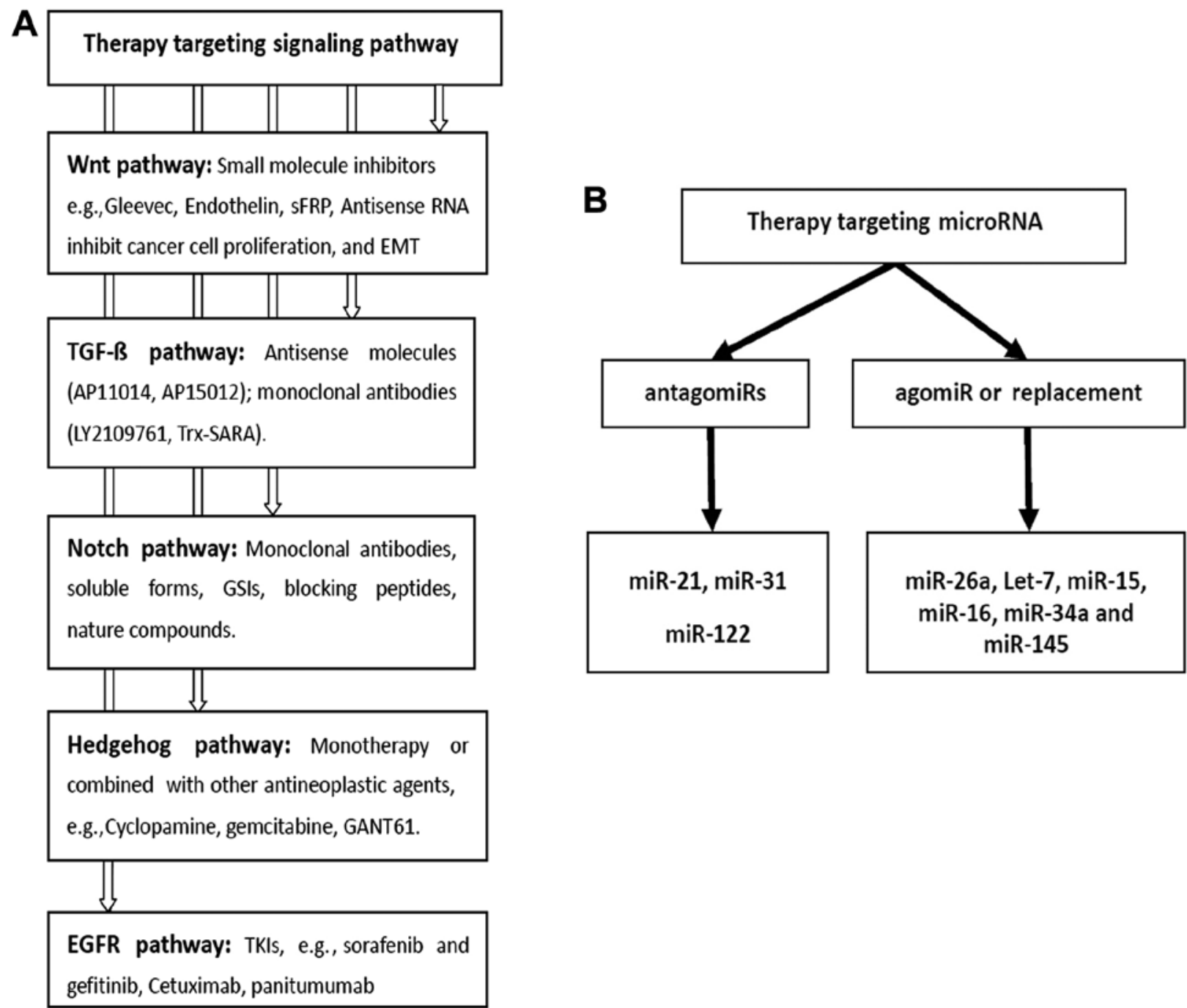

Figure 3. Targeting therapies in colorectal cancer cell lines. (A) Targeting point in the epithelial-mesenchymal transition-associated signaling pathway. (B) Restoration of silenced miRNAs and blocking of oncogenic miRNAs.

Drugs targeting Wnt/ $\beta$-catenin signaling pathway. The Wnt signaling pathway is closely associated with the occurrence and development of CRC. In vitro experiments on drugs targeting the Wnt signaling pathway are currently ongoing. Non-steroidal anti-inflammatory drugs (NSAIDs) including Aspirin (104), Sulindac (105), Celecoxib and rofecoxib (106) downregulate the $\beta$-catenin level by inducing $\beta$-catenin phosphorylation and degradation or inhibiting the transcription of target genes.

Small molecule inhibitor is a research hotspot on molecular targeting therapy. Gleevec, a type of tyrosine kinase inhibitor, is a small molecular protein, which can significantly inhibit CRC cell proliferation, reduce transcription activity, and induce $\beta$-catenin migration from the cell nucleus to the membrane (107). Endothelin (ET) can promote the degradation of $\beta$-catenin, inhibit target-gene cyclin D1 promoter activity, and induce cell-cycle arrest, apoptosis and tumor angiogenesis (108). The sFRP Wnt pathway inhibitor can suppress CRC cell proliferation and transformation ability (109). Antisense RNA can silence $\beta$-catenin expression or inhibit CRC cell proliferation and differentiation by eradicating the $\mathrm{TCF} / \beta$-catenin complex in the Wnt pathways (108). Thus, the
Wnt signaling pathway is expected to offer a new effective method for the treatment of CRC.

Drugs targeting the TGF- $\beta$ signaling pathway. As we described earlier, the TGF- $\beta$ signaling pathway plays a key role in CRC EMT. Therapeutic strategies against TGF- $\beta$ are effective methods that are classified into the ligand, ligand-receptor and intracellular levers (110). Powerful anti-TGF- $\beta$ strategies have been developed and tested in pre-clinical studies as well as clinical trials. Antisense molecules can prevent TGF- $\beta$ synthesis on the ligand lever by binding to specific mRNA. AP11014 and AP15012 are antisense molecules used in pre-clinical trials for treatment of non-small cell lung cancer, prostate carcinoma, CRC and MM, respectively (111). By blocking downstream signaling, monoclonal-neutralizing antibodies acting as receptor kinase inhibitors were more efficient as compared to ligand traps or antisense molecules (110). LY2109761 is a small molecule inhibiting the kinase activity of T $\beta R$ I and $\mathrm{T} \beta \mathrm{R}$ II. This compound inhibits metastatic formation in mouse models of breast cancer, CRC and pancreatic cancer (112-114). The Trx-SMAD anchor for receptor activation (Trx-SARA) is an example of a peptide aptamer, which reduces the levers of TGF- $\beta$-induced SMAD- $2 /-3$ in the complex (115), and inhibits 
EMT following TGF- $\beta$ stimulation in breast cancer epithelial cells (116). Ongoing pre-clinical trials are currently focused on other drugs targeting the TGF- $\beta$ signaling pathway. Future studies on drug development targeting EMT in CRC remain to be conducted.

Drugs targeting Notch signaling pathway. The Notch pathway has great potential as a new cancer treatment target. Various Notch inhibitors, including monoclonal antibodies against Notch receptors or ligands, soluble forms of the extracellular domain of Notch receptors or ligands, GSIs, blocking peptides, and nature compounds have been identified (117-124). GSIs are the most widely studied Notch receptors, having potential for bio-distribution and pan-Notch inhibition (125). GSIs are utilized in Phase I clinical trials as a monotherapy or in combination with other drugs (126). Therefore Notch is a potential antitumor therapeutic target and its implementation should occur subsequent to in-depth study with a focus on the Notch signaling pathway.

Drugs targeting Hedgehog signaling pathway. Currently, all of the small-molecules Hedgehog (Hh) pathway therapeutics in clinical trials are designed to target Smoothened (SMO). The reagents act as monotherapy or are combined with other antineoplastic agents for the treatement of various tumors including colon cancer. The $\mathrm{Hh}$ inhibitor cyclopamine is an antagonist of the Hh signaling pathway component SMO. Studies have shown that cyclopamine can eliminate pancreatic cancer metastasis (127). Cyclopamine inhibits the invasion of pancreatic cancer by suppressing EMT, upregulating E-cadherin and downregulating Snail. In an orthotopic xenograft model, the combination of cyclopamine and gemcitabine can restore metastases (128). GLI was found to be activated by other signaling pathways, such as Ras, TGF- $\beta$ and Wnt signaling pathways. In human colon carcinoma cells, specific targeting of GLI, which is downstream of SMO, can induce cancer cell death (129). GLI has a broader role, blocking the downstream gene transcription and activation (130-131).

Drugs targeting the EGFR signaling pathway. The epidermal growth factor receptor (EGFR) is known to be overexpressed in CRC EMT, while anti-EGFR therapy is considered effective. Tyrosine kinase inhibitors (TKIs), such as sorafenib and gefitinib, were designed to block the cascade of reactions which are crucial to tumor development and survival (132). Sorafenib is currently being tested in phase II trials of CRC. A recent study has indicated that the ability of TKIs strongly suppress P13K signaling in CRC, suggesting that such treatments have great therapeutic value (133). Cetuximab and panitumumab, monoclonal antibodies to EGFR, were approved by the FDA for the treatment of CRC in 2004 and 2006, respectively. The two antibodies prevent EGFR auto-phosphorylation by binding to the extracellular domain. They also inhibit activation of the MAPK and P13K downstream cell signaling pathways (132). In addition, the two drugs exhibited significant antitumor activity, although limitations and side effects were observed (134-137).

Targeting miRNA in CRC EMT. miRNAs play a key role in proliferation, cell cycle, evasion of apoptosis, invasion, migration and EMT. One of the most appealing properties of miRNA as therapeutic drugs in comparison with targeting single genes is their ability to target multiple molecules in the network. Abnormal expression levels of miRNAs show that, miRNAs can be used as drugs in CRC. Aiming at an abnormally high expression of onco-miR in tumor, small molecule drugs known as 'antagomiRs' have been designed, to knock out abnormal miRNAs or suppress expression of miRNAs. Targeting low or no expression of tumor-supressor miRNAs, corresponding exogenous miRNAs are known as 'agomiRs'. AgomiRs can be guided into the patient's body, restoring or improving the function of miRNAs in tissues (138).

Strategies for antagomiRs

Anti-miRNA oligonucleotides. Anti-miRNA oligonucleotides (AMOs) are single-stranded oligonucleotides consisting of 22-25 nucleotides, inhibiting miRNAs $(139,140)$. Because of not being chemically decorated, antisense oligomeric nucleotide chain is not stable, almost invalid in the body. AMOs are often modified chemically, such as 2'-O-methylation (141), 2'-O-methoxyl ethylation and locked nucleic acids (LNAs) $(142,143)$. The modification of AMOs can suppress miRNAs effectively. Experimental results showed that injecting inhibitors in mice specifically silenced their target miR-122 or miR-16 in liver and other organs, such as kidney, lung, heart and colon etc. (144). LNA-modified anti-miRNA oligonucleotides have been shown to inhibit miR-21 in breast cancer and in glioblastoma cells, resulting in the inhibition of tumor cells in vitro and in vivo. In xenograft tumor models, 2'-O-methyl-modified oligonucleotides (anti-miR-21) were injected into MCF-7 cells as instantaneous transfection. The results showed that the transfected tumor cells were reduced by $\sim 50 \%$. The study proved that 2'-O-methyl-modified oligonucleotides were a potential cancer treatment candidate $(145,146)$. Of note, inhibition of miR-21 expression in extrahepatic cholangiocarcinoma cells can increase the drug sensitivity of gemcitabine (147). Studies showed that the miRNA-based antitumor treatment combined with chemotherapy can improve treatment efficiency. However, the mechanism of downregulating the miRNA activity through AMOs inhibitors remains to be elucidated. The mechanism may occur by blocking miRNA biosynthesis via interaction with prior pre-miRNAs, or binding to mature miRNA to exert its role in the RISC compounds.

Other novel inhibitions. miRNAs sponges, miRNA-masking antisense oligodeoxynucleotides and small molecule inhibitors also suppress the overexpression of miRNA. Synthetized chemically, miRNAs sponges is a single-stranded RNA (148), blocking interactions between endogenous miRNA and its target mRNA (149). The sponge-mediated inhibition of the anti-metastatic miR-31 has been successfully applied in an orthotopic breast-cancer model. Results of that study demonstrated that a significant induction of lung metastases appeared, even with use of non-aggressive breast cancer cells (138-150). miRNA-masking antisense oligodeoxynucleotides bind perfectly to sites of complementary sequence, forming dimers with higher affinity, closing the sites of miRNAs through prior combining with target mRNA, and inhibiting the combination of miRNA and target mRNA (151). Other miRNA inhibitors, such as azobenzene, can block the transcription of miR-21, providing evidence that small organic molecules can be used in the miRNA context as well (152).

Strategies for agomiR or replacement. Treatment strategies, such as targeting low expression of miRNAs in CRC EMT, replacement therapy or agomiRs to restore physiological 
miRNA levels, can be applied. Current replacement therapy includes gene therapy and transfection of miRNA mimics. The approach of gene therapy involves generation of the pre-miRNA hairpin structure, transfecting and ensuring longterm and stable expression of mature miRNA. In addition, miRNA mimics, small double-stranded RNAs synthetized chemically, are widely used. It can stimulate active endogenous miRNA molecular and target-specific mRNA through transfection in vitro or in systemic administration in vivo by liposomes or nanoparticles polymer. Through virus-mediated miRNA re-introduction of miR-26a in liver cancer mouse, investigators observed the inhibition of cancer cell proliferation and induction of tumor-specific apoptosis (153). In vitro, Let-7 inhibits cancer cell growth in lung cancer cells (154). In an autochthonous NSCLC mouse model, the overexpression of Let-7 through lentiviral vector can lead to a significant growth reduction (155). Another example of miRNAs replacement therapy is miR-15 and miR-16, targeting apoptotic inhibition gene $\mathrm{Bcl}-2$. The re-introduction of miR-15a/miR-16 into prostate carcinoma xenografts by lentiviruses can also reduce tumor growth (156). In addition, using lipid-based delivery reagents, miR-34 led to the inhibition of non-small lung cancer and reduction of metastatic tumor load in the lung, basing on survivin inhibition $(157,158)$. The expression lever of miR-145, and downregulation in tumor tissues of CRC patients were increased in vitro. Thus, cell proliferation was reduced while sensitivity to radiotherapy was increased (159).

miRNAs and antisense miRNA drugs are currently at the pre-clinical stage. Owing to poor stability, low efficiency of transmission and toxic reaction, the clinical application of targeting miRNA requires additional investigation.

\section{Conclusion and perspective}

In the present review, we attempted to summarize the molecules, mechanisms and corresponding targeted therapies involved in the EMT process. In the past few years, it has become clear that EMT can reprogram normal epithelial cells. The process occurs systematically at the transcriptional, post-transcriptional, translational and post-translational levels. An improved understanding of the EMT process in CRC may lead to the identification of novel drug-targeted therapies and approaches.

\section{Acknowledgements}

This study was supported by a grant from the Shanghai Science and Technology Department of Medicine guiding biomedical projects (no. 134119a1400) and a grant from the Research Fund for the Doctoral Program of Higher Education (New Teacher) (no. 20130071120048).

\section{References}

1. Siegel R, Ma J, Zou Z and Jemal A: Cancer Statistics, 2014. CA Cancer J Clin 64: 9-29, 2014.

2. Thiery JP: Epithelial-mesenchymal transitions in development and pathologies. Curr Opin Cell Biol 15: 740-746, 2003.

3. Croce CM and Iorio MV: MicroRNA dysregulation in cancer: diagnostics, monitoring and therapeutics. EMBO Mol Med 4: 143-159, 2012.

4. Jeff JH and Yang J: Epithelial-mesenchymal plasticity in carcinoma metastasis. Genes Dev 27: 2192-2206, 2013.
5. Thiery JP and Sleeman JP: Complex networks orchestrate epithelial-mesenchymal-transitions. Nat Rev Mol Cell Biol 7: 131-142, 2006.

6. Jang J and Weinberg RA: Epithelial-mesenchymal transition: at the crossroads of development and tumor metastasis. Dev Cell 14: 818-829, 2008.

7. Pino MS, Kikuchi H, Zeng M, et al: Epithelial to mesenchymal transition is impaired in colon cancer cells with microsatellite instability. Gastroenterology 138: 1406-1417, 2010.

8. Savagner P: The epithelial-mesenchymal transition phenomenon. Ann Oncol 21: 89-92, 2010.

9. Bonnomet A, Brysse A, Tachsidis A, et al: Epithelial-tomesenchymal transitions and circulating tumor cells. J Mammary Gland Biol Neoplasia 15: 261-273, 2010.

10. Lun $\mathrm{H}$ and Shourong S: EMT phenomenon and related microRNAs in the malignant progression of tumor. Chin Oncol 21: 725-730, 2011.

11. Cavallaro U, Schaffhauser B, and Christofori G: Cadherins and the tumour progression: is it all in a switch? Cancer Lett 176: 123-128, 2002.

12. Saito Y, Takazawa H, Uzawa K, et al: Reduced expression of E-cadherin in oral squamous cell carcinoma: Relationship with DNA methylation of 5' CpG island. Int J Oncol 12: 293-298, 1998.

13. Lester RD, Jo M, Montel V, et al: uPAR induces epithelial-mesenchymal transition in hypoxic breast cancer cells. J Cell Biol 178: 425-436, 2007.

14. Berx G and Van Roy F: The E-cadherin/catenin complex: an important gatekeeper in breast cancer tumorigenesis and malignant progression. Breast Cancer Res 3: 289-293, 2001.

15. Canel M, Serrels A, Frame MC, et al: E-cadherin-integrin crosstalk in cancer invasion and metastasis. J Cell Sci 126: 393-401, 2013.

16. Derycke LD and Bracke ME: N-cadherin in the spotlight of cell-cell adhesion, differentiation, embryogenesis, invasion and signaling. Int J Dev Biol 48: 463-476, 2004.

17. Hazan RB, Qiao R, Keren R, et al: Cadherin switch in tumor progression. Ann NY Acad Sci 1014: 155-163, 2004.

18. Rosivatz E, Becker I, Bamba M, et al: Neoexpression of $\mathrm{N}$-cadherin in E-cadherin positive colon cancers. Int $\mathrm{J}$ Cancer 111: 711-719, 2004.

19. Colomiere M, Findlay J, Ackland L and Ahmed N: Epidermal growth factor-induced ovarian carcinoma cell migration is associated with JAK2/STAT3 signals and changes in the abundance and localization of alpha6betal integrin. Int J Biochem Cell Biol 41: 1034-1045, 2009.

20. Vora HH, Patel NA, Rajvik KN, et al: Cytokerain and vimentin expression in breast cancer. Int J Biol Markers 24: 38-46, 2009.

21. Shirahata A, Sakata M, Sakuraba K, et al: Vimentin methylation as a marker or advanced colorectal carcinoma. Anticancer Res 29: 279-281, 2009.

22. Wei J, Xu G, Wu M, et al: Overexpression of vimentin contributes to prostate cancer invasion and metastasis via src regulation. Anticancer Res 28: 327-334, 2008.

23. Pankov R and Yamada KM: Fibronectin at a glance. J Cell Science 115: 3861-3863, 2002.

24. Birchler MT, Milisavlijevic D, Pfaltz M, et al: Expression of the extra domain B of fibronectin, a marker of angiogenesis, in head and neck tumors. Laryngoscope 113: 1231-1237, 2003.

25. Cano A, Perez-Moreno MA, Rodrigo I, et al: The transcription factor snail controls epithelial-mesenchymal transitions by repressing E-cadherin expression. Nat Cell Biol 12: 76-83, 2000.

26. Hajra KM, Chen DY and Fearon ER: The SLUG zinc-finger protein represses E-cadherin in breast cancer. Cancer Res 62: 1613-1618, 2002.

27. Eger A, Aigner K, Sonderegger S, et al: DeltaEF1 is a transcriptional repressor of E-cadherin and regulates epithelial plasticity in breast cancer cells. Oncogene 24: 2375-2385, 2005.

28. Kim T, Veronese A, Pichiorri F, et al: p53 regulates epithelialmesenchymal transition through microRNAs targeting ZEB1 and ZEB2. J Exp Med 208: 875-883 2011.

29. Yang J, Mani SA, Donaher JL, et al: Twist, a master regulator of morphogenesis, plays an essential role in tumor metastasis. Cell 117: 927-939, 2004

30. Fang X, Cai Y, Liu J, et al: Twist2 contributes to breast cancer progression by promoting an epithelial-mesenchymal transition and cancer stem-like cell self-renewal. Oncogene 30: 4707-4720, 2011.

31. Casas E, Kim J, Bendesky A, et al: Snail2 is an essential mediator of Twist1-induced epithelial mesenchymal transition and metastasis. Cancer Res 71: 245-254, 2011. 
32. Nusse R and Varmus HE: Many tumors induced by the mouse mammary tumor virus contain a provirus integrated in the same region of the host genome. Cell 31: 99-109, 1982.

33. Kurayoshi M, Oue N, Yamamoto H, et al: Expression of Wnt-5a is correlated with aggressiveness of gastric cancer by stimulating cell migration and invasion. Cancer Res 66: 10439-10448, 2006.

34. Dissanayake SK, Wade M, Johnson CE, et al: The Wnt5A/protein kinase $\mathrm{C}$ pathway mediates motility in melanoma cells via the inhibition of metastasis suppressors and initiation of an epithelia to mesenchymal transition. J Biol Chem 282: 17259-17271, 2007

35. Huelsken $\mathbf{J}$ and Behrens J: The Wnt signaling pathway. J Cell Sci 115: 3977-3978, 2002.

36. King TD, Zhang W, Suto MJ, et al: Frizzled7 as an emerging target for cancer therapy. Cell Signal 24: 846-851, 2012.

37. MacDonald BT, Tamai K and He X: Wnt/beta-catenin signaling: components, mechanisms, and diseases. Dev Cell 17: 9-26, 2009.

38. Deka J, Wiedemann N, Anderle P, et al: Bcl9/Bcl9l are critical for Wnt-mediated regulation of stem cell traits in colon epithelium and adenocarcinomas. Cancer Res 70: 6619-6628, 2010

39. Hlubek F, Spaderna S, Schmalhofer O, et al: Wnt/FZD signaling and colorectal cancer morphogenesis. Front Biosci 12: 458-470, 2007.

40. Beiter K, Hiendlmeyer E, Brabletz T, et al: Beta-catenin regulates the expression of tenascin-C in human colorectal tumors Oncogene 24: 8200-8204, 2005.

41. Katsuno Y, Lamouille $S$ and Derynck R: TGF- $\beta$ signaling and epithelial-mesenchymal transition in cancer progression. Curr Opin Oncol 25: 76-84, 2013.

42. Watanabe Y, Itoh S, Goto T, et al: TMEPAI, a transmembrane TGF-beta-inducible protein, sequesters Smad proteins from active participation in TGF-beta signaling. Mol Cell 37: 123-134, 2010.

43. Shi Y and Massague J: Mechanisms of TGF-beta signaling from cell membrane to the nucleus. Cell 113: 685-700, 2003.

44. Tsukazaki T, Chiang TA, Davison AF, et al: SARA, a FYVE domain protein that recruits Smad2 to the TGFbeta receptor. Cell 95: 779-791, 1998.

45. Müller N, Reinacher-Schick A, Baldus S, et al: SMAD4 induces the tumor suppressor E-cadherin and P-cadherin in colon carcinoma cells. Oncogene 21: 6049-6058, 2002.

46. Mu Y, Gudey SK and Landström M: Non-SMAD signaling pathways. Cell Tissue Res 347: 11-20, 2012.

47. Hong M, Wilkes MC, Penheiter SG, et al: Non-Smad transforming growth factor- $\beta$ signaling regulated by focal adhesion kinase binding the p85 subunit of phosphatidylinositol 3-kinase. J Biol Chem 286: 17841-17850, 2011.

48. Kang JS, Liu C and Derynck R: New regulatory mechanisms of TGF-beta receptor function. Trends Cell Biol 19: 385-394, 2009.

49. Hartsough MT and Mulder KM: Transforming growth factor beta activation of $\mathrm{p} 44 \mathrm{mapk}$ in proliferating cultures of epithelial cells. J Biol Chem 270: 7117-7124, 1995.

50. Park SS, Eom YW, Kim EH, et al: Involvement of c-Src kinase in the regulation of TGF-betal-induced apoptosis. Oncogene 23 : 6272-6281, 2004.

51. Yu L, Hebert MC and Zhang YE: TGF-beta receptor-activated p38 MAP kinase mediates Smad-independent TGF-beta responses. EMBO J 21: 3749-3759, 2002.

52. Timmerman LA, Grego Bessa J, Raya A, et al: Notch promotes epithelial-mesenchymal transition during cardiac development and oncogenic transformation. Genes Dev 18 99-115, 2004.

53. Leong KG, Niessen K, Kulic I, et al: Jagged 1-mediated Notch activation induces epithelial-to-mesenchymal transition through Slug-induced repression of E-cadherin. J Exp Med 204: 2935-2948, 2007.

54. Sahlgren C, Gustafsson MV, Jin S, et al: Notch signaling mediates hypoxia induced tumor cell migration and invasion. Proc Natl Acad Sci USA 105: 6392-6397, 2008.

55. Veenendaal LM, Kranenburg O, Smakman N, et al: Differential Notch and TGFbeta signaling in primary colorectal tumors and their corresponding metastases. Cell Oncol 30: 1-11, 2008.

56. Murone M, Rosenthal A and de Sauvage FJ: Sonic hedgehog signaling by the patched-smoothened receptor complex. Curr Biol 9: 76-84, 1999.

57. Chen JS, Huang XH, Wang Q, et al: Sonic hedgehog signaling pathway induces cell migration and invasion through focal adhesion kinase/AKT signaling-mediated activation of matrix metalloproteinase (MMP)-2 and MMP-9 in liver cancer. Carcinogenesis 34: 10-19, 2013.
58. Zhang H, Berezov A, Wang Q, et al: ErbB receptors: from oncogenes to targeted cancer therapies. J Clin Invest 117: 2051-2058, 2007.

59. Oda K, Matsuoka Y, Funahashi A, et al: A comprehensive pathway map of epidermal growth factor receptor signaling. Mol Syst Biol 1: 2005.0010, 2005.

60. Wu L, Fan J and Belasco JG: MicroRNAs direct rapid deadenylation of mRNA. Proc Natl Acad Sci USA 103: 4034-4039, 2006

61. Wienholds E, Koudijs MJ, van Eeden FJ, et al: The microRNA-producing enzyme Dicerl is essencial for zebrafish development. Nat Genet 35: 217-218, 2003.

62. Lee RC, Feinbaum RL and Ambros V: The C. elegans heterochronic gene lin-4 encodes small RNAs with antisense complementarity to lin-14. Cell 75: 843-854, 1993.

63. Pasquinelli AE, Reinhart BJ, Slack F, et al: Conservation of the sequence and temporal expression of let-7 heterochronic regulatory RNA. Nature 408: 86-89, 2000.

64. Fang Y, Shi C, Manduchi E, et al: MicroRNA-10a regulation of proinflammatory phenotype in athero-susceptible endothelium in vivo and in vitro. Proc Natl Acad Sci USA 107: 13450-13455, 2010.

65. Tsai KW, Wu CW, Hu LY, et al: Epigenetic regulation of miR-34b and miR-129 expression in gastric cancer. Int J Cancer 129: 2600-2610, 2011

66. Yan W, Song G-X and Li Q: Advances in understanding the relationship between microRNAs and colorectal cancer. World Chin J Digestol 19: 3426-3431, 2011.

67. Ng EK, Chong WW, Jin H, et al: Differential expression of microRNAs in plasma of colorectal cancer patients: a potential marker for colorectal cancer screening. Gut 58: 1375-1381, 2009.

68. Lu J, Getz G, Miska EA, et al: MicroRNA expression profiles classify human cancers. Nature 435: 834-838, 2005.

69. Calin GA, Sevignani C, Dumitru CD, et al: Human microRNA genes are frequently located at fragile sites and genomic regions involved in cancers. Proc Natl Acad Sci USA 101: 2999-3004, 2004.

70. Hurteau GJ, Carlson JA, Spivack SD, et al: Overexpression of the microRNA has-miR-200c leads to reduced expression of transcription factor 8 and increased expression of E-cadherin. Cancer Res 67: 7972-7976, 2007.

71. Gregory PA, Bert AG, Paterson EL, et al: The miR-200 family and miR-205 regulate epithelial to mesenchymal transition by targeting ZEB1 and SIP1. Nat Cell Biol 10: 593-601, 2008.

72. Park SM, Gaur AB, Lengyel E, et al: The miR-200 family determines the epithelial phenotype of cancer cells by targeting the E-cadherin repressors ZEB1 and ZEB2. Genes Dev 22: 894-907, 2008.

73. Burk U, Schubert J, Wellner U, et al: A reciprocal repression between ZEB1 and members of the miR-200 family promotes EMT and invasion in cancer cells. EMBO Rep 9: 582-589, 2008

74. Shell S, Park SM, Radjabi AR, et al: Let-7 expression defines two differentiation stages of cancer. Proc Natl Acad Sci USA 104: 11400-11405, 2007.

75. Liu X, Wang C, Chen Z, et al: MicroRNA-138 suppresses epithelial-mesenchymal transition in squamous cell carcinoma lines. Biochem J 440: 23-31, 2011.

76. Castilla MA, Moreno-Bueno G, Romero-Perez L, et al: Micro-RNA signature of the epithelial-mesenchymal transition in endometrial carcinosarcoma. J Pathol 233: 72-80, 2011.

77. Li QQ, Chen ZQ, Cao XX, et al: Involvement of NF- $\mathrm{BB} / \mathrm{miR}-448$ regulatory feedback loop in chemotherapy-induced epithelialmesenchymal transition of breast cancer cells. Cell Death Differ 18: 16-25, 2011

78. Meng Z, Fu X, Chen X, et al: miR-194 is a marker of hepatic epithelial cells and suppresses metastasis of liver cancer cells in mice. Hepatology 52: 2148-2157, 2010.

79. Ma L, Teruya-Feldstein J and Weinberg RA: Tumor invasion and metastasis initiated by microRNA-10b in breast cancer. Nature 449: 682-688, 2007.

80. Kong W, Yang H, He L, et al: MicroRNA155 is regulated by the transforming growth factor beta/Smad pathway and contributes to epithelial cell plasticity by targeting RhoA. Mol Cell Biol 28: 6773-6784, 2008.

81. Cottonham CL, Kaneko S and Xu L: miR-21 and miR-31 converge on TIAM1 to regulate migration and invasion of colon carcinoma cells. J Biol Chem 285: 35293-35302, 2010.

82. Asangani IA, Rasheed SA, Nikolova DA, et al: MicroRNA-21 (miR-21) post-transcriptionally downregulates tumor suppressor Pdcd4 and stimulates invasion, intravasation and metastasis in colorectal cancer. Oncogene 27: 2128-2136, 2008. 
83. Wang $\mathrm{P}$, Zho $\mathrm{F}$, Zheng $\mathrm{X}$, et al: microRNA-21 negatively regulates $\mathrm{Cdc} 25 \mathrm{~A}$ and cell cycle progression in colon cancer cells. Cancer Res 69: 8157-8165, 2009.

84. Ma L, Young J, Prabhala H, et al: miR-9, a MYC/MYCNactivated microRNA, regulates E-cadherin and cancer metastasis. Nat Cell Biol 12: 247-256, 2010.

85. Vetter G, Saumet A, Moes M, et al: miR-661 expression in SNAI1-induced epithelial to mesenchymal transition contributes to breast cancer cell invasion by targeting Nectin-1 and StarD10 messengers. Oncogene 29: 4436-4448, 2010.

86. Castilla MA, Moreno-Bueno G, Romero-Pérez L, et al: Micro-RNA signature of the epithelial-mesenchymal transition in endometrial carcinosarcoma. J Pathol 223: 72-80, 2011.

87.De Krijger I, Mekenkamp LJ, Punt CJ and Nagtegaal ID: MicroRNAs in colorectal cancer metastasis. J Pathol 224 438-447, 2011.

88. Liu $\mathrm{M}$ and Chen $\mathrm{H}$ : The role of microRNAs in colorectal cancer. J Genet Genomics 37: 347-358, 2010.

89. Spaderna S, Schmalhofer O, Hlubek F, et al: A transient EMT-linked loss of basement membranes indicates metastasis and poor survival in colorectal cancer. Gastroenterology 131: 830-840, 2006.

90. Spaderna S, Schmalhofer O, Wahlbuhl M, et al: The transcriptional repressor ZEB1 promotes metastasis and loss of cell polarity in cancer. Cancer Res 68: 537-544, 2008

91. Korpal M,Lee ES, Hu G and Kang Y: The miR-200 family inhibits epithelial-mesenchymal transition and cancer cell migration by direct targeting of E-cadherin transcriptional repressors ZEB1 and ZEB2. J Biol Chem 283: 14910-14914, 2008.

92. Brabletz S and Brabletz T: The ZEB/miR-200 feedback loop - a motor of cellular plasticity in development and cancer? EMBO Rep 11: 670-677, 2010.

93. Fujita S, Ito T, Mizutani T, et al: miR-21 gene expression triggered by AP-1 is sustained through a double-negative feedback mechanism. J Mol Biol 378: 492-504, 2008

94.Ferraro A, Kontos CK, Boni T, et al: Epigenetic regulation of miR-21 in colorectal cancer: ITGB4 as a novel miR-21 target and a three-gene network (miR-21-ITGB4-PDCD4) as predictor of metastatic tumor potential: Epigenetics 9: 129-141, 2014.

95. Bommer GT, Gerin I, Feng Y, et al: p53-mediated activation of miRNA34 candidate tumor-suppressor genes. Curr Biol 17: 1298-1307, 2007.

96. Chang TC, Wentzel EA, Kent OA, et al: Transactivation of miR-34a by p53 broadly influences gene expression and promotes apoptosis. Mol Cell 26: 745-752, 2007.

97. Yamakuchi M,Ferlito M and Lowenstein CJ: miR-34a repression of SIRT1 regulates apoptosis. Proc Natl Acad Sci USA 105: 13421-13426, 2008

98. Siemens H, Jackstadt R, Hünten S, et al: miR-34 and SNAIL form a double-negative feedback loop to regulate epithelialmesenchymal transitions. Cell Cycle 10: 4256-4271, 2011.

99. Kim NH, Kim HS, Li XY, et al: A p53/miRNA-34 axis regulates Snaill-dependent cancer cell epithelial-mesenchymal transition. J Cell Biol 195: 417-433, 2011

100. Rokavec M, Oner MG, Li H, et al: IL-6R/STAT3/miR-34a feedback loop promotes EMT-mediated colorectal cancer invasion and metastasis. J Clin Invest 124: 1853-1867, 2014.

101. Bandi N, Zbinden S, Gugger M, et al: miR-15a and miR16 are implicated in cell cycle regulation a Rb-dependent manner and are frequently deleted or down-regulated in non-small cell lung cancer. Cancer Res 69: 5553-5559, 2009.

102. Jackstadt R, Röh S, Neumann J, et al: AP4 is a mediator of epithelial-mesenchymal transition and metastasis in colorectal cancer. J Exp Med 210: 1331-1350, 2013.

103. Shi L, Jackstadt R, Siemens H, et al: p53-induced miR-15a/16-1 and AP4 form a double-negative feedback loop to regulate epithelial-mesenchymal transition and metastasis in colorectal cancer. Cancer Res 74: 532-542, 2014.

104.Dihlmann S, Siermann A and von Knebel Doeberitz M: The nonsteroidal anti-inflammatory drugs aspirin and indomethacin attenuate beta-catenin/TCF-4 signaling. Oncogene 20: 645-653, 2001.

105. Boon EM, Keller JJ, Wormhoudt TA, et al: Sulindac targets nuclear beta-catenin accumulation and Wnt signaling in adenomas of patients with familial adenomatous polyposis and in human colorectal cancer cell lines. Br J Cancer 90: 224-229, 2004

106. Linna L and Shoujun Y: Wnt/ $\beta$-catenin signaling pathway and strategy of originating and treatment in colorectal cancer. World Chin J Digestol 14: 201-206, 2006.
107.Zhou L, An N, Haydon RC, et al: Tyrosine kinase inhibitor STI-571/Gleevec down-regulatates the beta-catenin signaling activity. Cancer Lett 193: 161-170, 2003.

108. Green DW, Roh H, Pippin JA and Drebin JA: Beta-catenin antisense treatment decreases beta-catenin expression and tumor growth rate in colon carcinoma xenografts. J Surg Res 101: 16-20, 2001.

109. Suzuki H, Watkins DN, Jair KW, et al: Epigenetic inactivation of SFRP genes allows constitutive WNT signaling in colorectal cancer. Nat Genet 36: 417-422, 2004

110. Katz LH, Li Y, Chen JS, et al: Targeting TGF- $\beta$ signaling in cancer. Expert Opin Ther Targets 17: 743-760, 2013.

111. Lampropoulos P,Zizi-Sermpetzoglou A, Rizos S, et al: TGF-beta signaling in colon carcinogenesis. Cancer Lett 314: 1-7, 2012.

112. Melisi D, Ishiyama S, Sclabas GM, et al: LY2109761, a novel transforming growth factor beta receptor type I and type II dual inhibitor, as a therapeutic approach to suppressing pancteatic cancer metastasis. Mol Cancer Ther 7: 829-840, 2008.

113. Korpal M, Yan J, Lu X, et al: Imaging transforming growth factor-beta signaling dynamics and therapeutic response in breast cancer bone metastasis. Nat Med 15: 960-966, 2009.

114. Zhang B, Halder SK, Zhang S and Datta PK: Targeting transforming growth factor-beta signaling in liver metastasis of colon cancer. Cancer Lett 277: 114-120, 2009.

115. Connolly EC, Freimuth J and Akhurst RJ: Comlexities of TGF- $\beta$ targeted cancer therapy. Int J Biol Sci 8: 964-978, 2012.

116. Zhao BM and Hoffmann FM: Inhibition of transforming growth factor-beta1-induced signaling and epithelial-to-mesenchymal transition by the Smad-binding peptide aptamer Trx-SARA. Mol Biol Cell 17: 3819-3831, 2006.

117. Espinoza I, Pochampally R, Xing F, et al: Notch signaling: targeting cancer stem cells and epithelial-to-mesenchymal transition. Onco Targets Ther 6: 1249-1259, 2013.

118. Yan M and Plowman GD: Delta-like 4/Notch signaling and its therapeutic implications. Clin Cancer Res 13: 7243-7246, 2007.

119. Hayashi I, Takatori S, Urano Y, et al: Neutralization of the $\gamma$-secretase activity by monoclonal antibody against extracellular domain of nicastrin. Oncogene 31: 787-798, 2012.

120. Funahashi Y, Hernandez SL, Das I, et al: A notch1 ectodomain construct inhibits endothelial notch signaling, tumor growth, and angiogenesis. Cancer Res 68: 4727-4735, 2008.

121. Tammam J, Ware C, Efferson C, et al: Down-regulation of the Notch pathway mediated by a gamma-secretase inhibitor induces anti-tumor effects in mouse models of T-cell leukaemia. Br J Pharmacol 158: 1183-1195, 2009.

122. Fouladi M, Stewart CF, Olson J, et al: Phase I trial of MK_0752 in children with refractory CNS malignancies: a pediatric brain tumor consortium study. J Clin Oncol 29: 3529-3534, 2011.

123. Moellering RE, Cornejo M, Davis TN, et al: Direct inhibition of the NOTCH transcription factor complex. Nature 462: 182-188, 2009.

124.Zhou W, Kallifatidis G, Baumann B, et al: Dietary polyphenol quercetin targets pancreatic cancer stem cells. Int J Oncol 37: $551-561,2010$

125. Kallifatidis G, Labsch S, Rausch V, et al: Sulforaphane increases drug-mediated cytotoxicity toward cancer stem-like cells of pancreas and prostate. Mol Ther 19: 188-195, 2011.

126. Espinozal I and Miele L: Notch inhibitors for cancer treatment. Pharmacol Ther 139: 95-110, 2013.

127. Kelleher FC: Hedgehog signaling and therapeutics in pancreatic cancer. Carcinogenesis 32: 445-451, 2011.

128. Feldmann G, Dhara S, Fendrich V, et al: Blockade of Hedgehog signaling inhibits pancreatic cancer invasion and metastases: a new paradigm for combination therapy in solid cancers. Cancer Res 67: 2187-2196, 2007.

129. Agyeman A, Mazumdar T and Houghton JA: Regulation of DNA damage following termination of Hedgehog $(\mathrm{HH})$ survival signaling at the level of the GLI genes in human colon cancer. Oncotarget 3: 854-868, 2012.

130. Stecca B and Ruiz I Altaba A: Context-dependent regulation of the GLI code in cancer by Hedgehog and non-Hedgehog signals. J Mol Cell Biol 2: 84-95, 2010

131. Lauth M and Toftqard R: Non-canonical activation of GLI transcription factors: implications for targeted anti-cancer therapy. Cell Cycle 6: 2458-2463, 2007.

132. Hegan S, Orr MC and Doyle B: Targeted therapies in colorectal cancer - an integrative view by PPPM. EPMA J 4: 3, 2013.

133. Ebi H, Corcoran RB, Singh A, et al: Receptor tyrosine kinases exert dominant control over PI3K signaling in human KRAS mutant colorectal cancers. J Clin Invest 121: 4311-4321, 2011. 
134. Van Cutsem E, Peeters M, Siena S, et al: Open-label phase III trial of panitumumab plus best supportive care compared with best supportive care alone in patients with chemotherapyrefractory metastatic colorectal cancer. J Clin Oncol 25 1658-1664, 2007.

135.Jonker DJ, O'Callaghan CJ, Karapetis CS, et al: Cetuximab for the treatment of colorectal cancer. N Engl J Med 357: 2040-2048, 2007.

136. Van Cutsem E, Köhne CH, Lang I, et al: Cetuximab plus irinotecan, fluorouracil, and leucovorin as first-line treatmen for metastatic colorectal cancer: updated analysis of overall survival according to tumor KRAS and BRAF mutation status. J Clin Oncol 29: 2011-2019, 2011.

137. Heinemann V, von Weikersthal LF, Decker T, et al: Randomized comparison of FOLFIRI plus cetuximab versus FOLFIRI plus bevacizumab as first-line treatment of KRAS wild-type metastatic colorectal cancer: German AIO study KRK-0306 (FIRE-3). J Clin Oncol 31: LBA3506 ASCO Annual Meeting, 2013.

138. Dassow H and Aigner A: MicroRNAs (miRNAs) in colorectal cancer: from aberrant expression towards therapy. Curr Pharm Des 19: 1242-1252, 2013.

139. Weiler J, Hunziker J and Hall J: Anti-miRNA oligonucleotides (AMOs): ammunition to target miRNAs implicated in human disease. Gene Ther 13: 496-502, 2006.

140. Garzon R, Marcucci G and Croce CM: Targeting microRNAs in cancer: rationale, strategies and challenges. Nat Rev Drug Discov 9: 775-789, 2010

141. Yang Z, Vilkaitis G, Yu B, et al: Approaches for studying MicroRNA and small interfering RNA methylation in vitro and in vivo. Methods Enzymol 427: 139-154, 2007.

142. Ørom UA, Kauppinen S and Lund AH: LNA-modified oligonucleotides mediate specific inhibition of microRNA function Gene 10: 137-141, 2006.

143. Vester B and Wengel J: LNA (locked nucleic acid): high-affinity targeting of complementary RNA and DNA. Biochemistry 43: 13233-13241, 2004.

144. Krützfeldt J, Rajewsky N, Braich R, et al: Silencing of microRNAs in vivo with 'antagomirs'. Nature 438: 685-689, 2005.

145. Si ML, Zhu S, Wu H, et al: miR-21-mediated tumor growth. Oncogene 26: 2799-2803, 2007.

146. Corsten MF, Miranda R, Kasmieh R, et al: MicroRNA-21 knockdown disrupts glioma growth in vivo and displays synergistic cytotoxicity with neural precursor cell delivered S-TRAIL in human gliomas. Cancer Res 67: 8994-9000, 2007.
147. Meng F, Henson R, Lang M, et al: Involvement of human microRNA in growth and response to chemotherapy in human cholangiocarcinoma cell lines. Gastroenterology 130: 2113-2129, 2006.

148. Ebert MS, Neilson JR and Sharp PA: MicroRNA sponges: competitive inhibitors of small RNAs in mammalian cells. Nat Methods 4: 721-726, 2007.

149.Ebert MS and Sharp PA: MicroRNA sponges: progress and possibilities. RNA 16: 2043-2050, 2010.

150. Valastyan S, Reinhardt F, Benaich N, et al: A pleiotropically acting microRNA, miR-31, inhibits breast cancer metastasis. Cell 137: 1032-1046, 2009.

151. Xiao J, Yang B, Lin H, et al: Novel approaches for gene-specific interference via manipulating actions of microRNAs: examination on the pacemaker channel genes HCN2 and HCN4. J Cell Physiol 212: 285-292, 2007.

152. Gumireddy K, Young DD, Xiong X, et al: Small-molecule inhibitors of microrna miR-21 function. Angew Chem Int Ed Engl 47: 7482-7484, 2008

153. Lennox KA and Behlke MA: Chemical modification and design of anti-miRNA oligonucleotides. Gene Ther 18: 1111-1120, 2011.

154. Yang N, Kaur S, Volinia S, et al: MicroRNA microarray identifles Let-7i as a novel biomarker and therapeutic target in human epithelial ovarian cancer. Cancer Res 68: 10307-10314, 2008.

155.Kumar MS, Erkeland SJ, Pester RE, et al: Suppression of non-small cell lung tumor development by the let-7 microRNA family. Proc Natl Acad Sci USA 105: 3903-3908, 2008.

156. Bonci D, Coppola V, Musumeci M, et al: The miR-15a-miR-16-1 cluster controls prostate cancer by targeting multiple oncogenic activities. Nat Med 14: 1271-1277, 2008.

157. Wiggins JF, Ruffino L, Kelnar K, et al: Development of a lung cancer therapeutic based on the tumor suppressor microRNA-34. Cancer Res 70: 5923-5930, 2010.

158. Chen Y, Zhu X, Zhang X, et al: Nanoparticles modified with tumor-targeting scFv deliver siRNA and miRNA for cancer therapy. Mol Ther 18: 1650-1656, 2010.

159. La Rocca G, Badin M, Shi B, et al: Mechanism of growth inhibition by MicroRNA 145: the role of the IGF-1 receptor signaling pathway. J Cell Physiol 220: 485-491, 2009. 